\title{
LINEARISABLE MAPPINGS AND THE LOW-GROWTH CRITERION
}

\author{
A. Ramani \\ CPT, Ecole Polytechnique \\ CNRS, UMR 7644 \\ 91128 Palaiseau, France \\ B. Grammaticos \\ GMPIB, Université Paris VII \\ Tour 24-14, $5^{e}$ étage, case 7021 \\ 75251 Paris, France \\ S. LAFORTUNE ${ }^{\dagger}$ \\ LPTMC et GMPIB, Université Paris VII \\ Tour 24-14, $5^{e}$ étage, case 7021 \\ 75251 Paris, France \\ Y. Онта \\ Department of Applied Mathematics \\ Faculty of Engineering, Hiroshima University \\ 1-4-1 Kagamiyama, Higashi-Hiroshima, 739-8527 Japan
}

\begin{abstract}
We examine a family of discrete second-order systems which are integrable through reduction to a linear system. These systems were previously identified using the singularity confinement criterion. Here we analyse them using the more stringent criterion of nonexponential growth of the degrees of the iterates. We show that the linearisable mappings are characterised by a very special degree growth. The ones linearisable by reduction to projective systems exhibit zero growth, i.e. they behave like linear systems, while the remaining ones (derivatives of Riccati, Gambier mapping) lead to linear growth. This feature may well serve as a detector of integrability through linearisation.

$\dagger$ Permanent address: CRM, Université de Montréal, Montréal, H3C 3J7 Canada
\end{abstract}


Integrability of discrete systems is a concept that can be understood on the basis of our experience on integrable continuous systems. The progress accomplished in the domain of discrete systems this last decade has made possible the identification of the possible types of integrability. The parallel with continuous systems is almost perfect. Three main types of integrable discrete systems seem to exist [1]:

a) Systems which possess a sufficient number of constants of motion. The QRT family of mappings [2] is a nice example of such a system.

b) Systems which can be reduced to linear mappings. They will be examined in detail in this paper.

c) Systems which can be obtained as the compatibility condition for some linear system i.e. systems that possess a Lax pair. Nice examples of such systems are the discrete Painlevé equations [3]. Given the Lax pair one can reduce the integration of the nonlinear mapping to the solution of an isomonodromy problem.

It is clear that the integration of a given integrable discrete system may proceed along any of the lines sketched above. One can, for example, perform one first integration using a constant of motion whereupon the system becomes linearisable and so on.

The very existence of integrable mappings (and their relative rarity) made their detection particularly interesting. Integrability detectors must, of course, be based on the properties which are characteristic of integrability. In this spirit we have proposed the singularity confinement property [4] based on the observation that a singularity spontaneously appearing in an integrable mapping disappears after some iterations: it is "confined" in the sense that it does not propagate ad infinitum. The singularity confinement criterion is a necessary one for integrability but, as we have already remarked in [1], it is not sufficient. This was explained in ample details by Hietarinta and Viallet [5] who have proposed the notion of algebraic entropy as a stronger criterion which could well be sufficient. This criterion is based on the ideas of Arnold [6] and Veselov [7] on the growth of the degrees of the iterates of some initial data under the action of the mapping. The main argument is that a generic, nonintegrable mapping has an exponential degree growth, while integrability is associated with low growth, typically polynomial. Although the degree itself is not invariant under coordinate changes, the type of growth, as pointed out by Bellon and Viallet [8], is invariant. The authors of [5] and [8] have introduced the notion of algebraic entropy defined as $E=\lim _{n \rightarrow \infty}\left(\log d_{n}\right) / n$, where $d_{n}$ is the degree of the $n$th iterate. Generic, nonintegrable mappings have nonzero algebraic entropy. The conjecture is that integrability, associated to polynomial growth, leads to zero algebraic entropy. In [9] we have examined the results on discrete Painlevé equations based on the singularity confinement criterion in the light of the low-growth approach. Our main finding was that singularity confinement is 
sufficient in order to deautonomize a given integrable autonomous mapping. This result led to the proposal of a dual approach for the study of discrete integrability based on the successive applications on the singularity confinement and low-growth criteria, the latter being implemented only after the first is used to simplify the problem down to tractable proportions.

The aim of this paper is to examine this particular class of mappings which are linearisable and study their growth properties. Most of these systems were obtained using the singularity confinement criterion and thus a study of the growth of the degree of the iterates would be an interesting complementary information. Moreover, as we will show, the linearisable systems do possess particular growth properties which set them apart from the other integrable discrete systems.

The first mapping we are going to treat is a two-point mapping of the form $x_{n+1}=f\left(x_{n}, n\right)$ where $f$ is rational in $x_{n}$ and analytical in $n$. In [1] we have shown that for all $f$ 's of the form $\sum_{i} \frac{\alpha_{i}}{\left(x+\beta_{i}\right)^{\nu_{i}}}$ the singularity confinement requirement is satisfied. However all those mappings cannot be integrable: the discrete Riccati, $x_{n+1}=\alpha+\frac{\lambda}{x_{n}+\beta}$, is the only expected integrable one. Our argument in [1], for the rejection of these confining but nonintegrable cases, was based on the proliferation of the preimages of a given point. If we solve the mapping for $x_{n}$ in terms of $x_{n+1}$ we do not find a uniquely defined $x_{n}$ and, iterating, the number of $x_{n-k}$ grows exponentially. In what follows we shall analyse this two-point mapping in the light of the algebraic entropy approach. We start from the simplest case which we expect to be nonintegrable,

$$
x_{n+1}=\alpha+\frac{\lambda}{x_{n}+\beta}+\frac{\mu}{x_{n}+\gamma} .
$$

The initial condition we are going to iterate is $x_{0}=p / q$ and the degree we calculate is the homogeneous degree in $p$ and $q$ of the numerator (or the denominator) of the iterate. We obtain readily the following degree sequence $d_{n}=1,2,4,8,16, \ldots$ i.e. $d_{n}=2^{n}$. Thus the algebraic entropy of the mapping is $\log (2)>0$, an indication that the mapping cannot be integrable. In the present case it was quite easy to guess an analytical expression for the degree. What we do in general in order to obtain a closed-form expression for the degrees of the iterates, is to compute a sufficient number of them. Then we establish heuristically an expression of the degree, compute the next few ones and check that they agree with the analytical expression prediction. Now we ask how can one curb the growth and make it nonexponential. It turns out that the only possibilities are $\lambda \mu=0$ or $\beta=\gamma$. In either case mapping (1) becomes a homography. The degree in this case is simply $d_{n}=1$ for all $n$. This is an interesting result, clearly due to the fact that the homographic mapping is linearisable through a simple Cole-Hopf transformation. 
The second mapping we shall examine is one due to Bellon and collaborators [10]

$$
\begin{aligned}
& x_{n+1}=\frac{x_{n}+y_{n}-2 x_{n} y_{n}^{2}}{y_{n}\left(x_{n}-y_{n}\right)}, \\
& y_{n+1}=\frac{x_{n}+y_{n}-2 x_{n}^{2} y_{n}}{x_{n}\left(y_{n}-x_{n}\right)} .
\end{aligned}
$$

The degree growth in this case is studied starting from $x_{0}=r, y_{0}=p / q$ and again we calculate the homogeneous degree of the iterate in $p$ and $q$, i.e. we set the degree of $r$ to zero. (Other choices could have been possible but the conclusion would not depend on these details.) We obtain the degrees $d_{x_{n}}=0,2,2,4,4,6,6, \ldots$ and $d_{y_{n}}=1,1,3,3,5,5, \ldots$ i.e. a linear degree-growth. This is in perfect agreement with the integrable character of the mapping. As was shown in [11] it does satisfy the unique preimage requirement and possesses a constant of motion $k=\frac{1-x_{n} y_{n}}{y_{n}-x_{n}}$, the use of which reduces it to a homographic mapping for $x_{n}$ or $y_{n}$.

The third mapping we are going to study is the one proposed in [1]

$$
\begin{gathered}
x_{n+1}=\frac{x_{n}\left(x_{n}-y_{n}-a\right)}{x_{n}^{2}-y_{n}}, \\
y_{n+1}=\frac{\left(x_{n}-y_{n}\right)\left(x_{n}-y_{n}-a\right)}{x_{n}^{2}-y_{n}}
\end{gathered}
$$

where $a$ was taken constant. We start by assuming that $a$ is an arbitrary function of $n$ and compute the growth of the degree. We find $d_{x_{n}}=0,1,2,3,4,5,6,7,8, \ldots$ and $d_{y_{n}}=$ $1,2,3,4,5,6,7,8,9, \ldots$ i.e. again a linear growth. This is an indication that (3) is integrable for arbitrary $a_{n}$ and indeed it is. Dividing the two equations we obtain $y_{n+1} / x_{n+1}=$ $1-y_{n} / x_{n}$ i.e. $y_{n} / x_{n}=1 / 2+k(-1)^{n}$ whereupon (3) is reduced to a homographic mapping for $x$. Thus in this case the degree-growth has succesfully predicted integrability.

A picture starts emerging at this point. While in our study of discrete Painlevé equations and the QRT mapping we found quadratic growth of the degree of the iterate, linearisable second-order mappings seem to lead to slower growth. In order to investigate this property in detail we shall analyse the three-point mapping we have studied in $[12,13]$ from the point of view of integrability in general and linearisability in particular. The generic mapping studied in [13] was one trilinear in $x_{n}, x_{n+1}, x_{n-1}$. Several cases were considered. Our starting point is the mapping,

$$
x_{n+1} x_{n} x_{n-1}+\beta x_{n} x_{n+1}+\zeta \eta x_{n+1} x_{n-1}+\gamma x_{n} x_{n-1}+\beta \gamma x_{n}+\eta x_{n-1}+\zeta x_{n+1}+1=0 .
$$

We start with the initial conditions $x_{0}=r, x_{1}=p / q$ and compute the homogeneous degree in $p, q$ at every $n$. We find $d_{n}=0,1,1,2,3,5,8,13, \ldots$ i.e. a Fibonacci sequence $d_{n+1}=$ 
$d_{n}+d_{n-1}$ leading to exponential growth of $d_{n}$ with asymptotic ratio $\frac{1+\sqrt{5}}{2}$. Thus mapping (4) is not expected to be integrable in general. However, as shown in [13] integrable subcases do exist. We start by requiring that the degree growth be less rapid and as a drastic decrease in the degree we demand that $d_{3}=1$ instead of 2 . We find that this is possible when either $\beta=\zeta=0$ in which case the mapping reduces to:

$$
x_{n+1}=-\gamma-\frac{\eta}{x_{n}}-\frac{1}{x_{n} x_{n-1}}
$$

or $\gamma=\eta=0$, giving a mapping identical to (5) after $x \rightarrow 1 / x$. In this case the degree is $d_{n}=1$ for $n>0$. Equation (5) is the generic linearisable three-point mapping, written in canonical form. Its linearisation can be obtained in terms of a projective system [13] i.e. a system of three linear equations, a fact which explains the constancy of the degree.

The trilinear three-point mapping possesses also many nongeneric subcases, some of which are integrable. The first nongeneric case writes:

$$
x_{n}\left(\gamma x_{n-1}+\epsilon\right)+\left(x_{n+1}+1\right)\left(\eta x_{n-1}+1\right)=0 .
$$

The degrees of the iterates of mapping (6) form again a Fibonacci sequence even in the case $\epsilon=0$ or $\eta=0$. The only case that presents a slightly different behaviour is the case $\gamma=0$ :

$$
\left(x_{n+1}+1\right)\left(\eta x_{n-1}+1\right)+\epsilon x_{n}=0 .
$$

In the generic case the degree of the iterate behaves like $d_{n}=0,1,1,1,2,2,3,4,5,7$, $9,12,16,21,28,37,49, \ldots$ satisfying the recursion relation $d_{n+1}=d_{n-1}+d_{n-2}$ leading to an exponential growth with asymptotic ratio $\left(\frac{1}{2}+\sqrt{\frac{23}{108}}\right)^{1 / 3}+\left(\frac{1}{2}-\sqrt{\frac{23}{108}}\right)^{1 / 3}$. Although the mapping is generically nonintegrable it does possess integrable subcases. Requiring for example that $d_{4}=1$ we obtain the constraint $\epsilon=\eta=1$ and the mapping becomes periodic with period 5 . If we require $d_{5}=1$, we obtain $\epsilon=-\eta_{n+1}\left(\eta_{n}-1\right)$ and $\eta_{n+1} \eta_{n} \eta_{n-1}-\eta_{n+1} \eta_{n}+\eta_{n+1}-1=0$, leading again to a periodic mapping with period 8. In these cases, the degree of the iterates exhibits, of course, a periodic behaviour. A more interesting result is obtained if we require $d_{9}<7$. We find that the condition $\eta=1$ and $\epsilon$ an arbitrary constant leads to a nonexponential degree growth $d_{n}=$ $0,1,1,1,2,2,3,4,5,6,7,9,10,12,14,15,18,20,22,25,27,30,33,36,39,42,46,49, \ldots$ Although the detailed behaviour of $d_{n}$ is pretty complicated one can see that the growth is quadratic: we have, for example, $d_{4 m+1}=m(m+1)$ for $m>0$. Thus this mapping is expected to be integrable and indeed, it is a member of the QRT family. Its constant of motion is given by

$$
K=y_{n+1}+y_{n}-\epsilon\left(\frac{y_{n+1}}{y_{n}}+\frac{y_{n}}{y_{n+1}}\right)+\epsilon(\epsilon+1)\left(\frac{1}{y_{n}}+\frac{1}{y_{n+1}}\right)-\frac{\epsilon^{2}}{y_{n} y_{n+1}}
$$


where $y_{k}=x_{k}+1$. The second nongeneric case is:

$$
\gamma x_{n} x_{n-1}+\delta x_{n+1} x_{n-1}+\epsilon x_{n}+\zeta x_{n+1}=0 .
$$

A study of the degree-growth leads always to exponential growth with asymptotic ratio $\frac{1+\sqrt{5}}{2}$, except when $\gamma=0$ in which case the degrees obey the recurrence $d_{n+1}=d_{n-1}+d_{n-2}$. No integrable subcases are expected for mapping (8). The last nongeneric case we shall examine is

$$
\gamma x_{n} x_{n-1}+x_{n+1} x_{n-1}+\epsilon x_{n}+\eta x_{n-1}=0 .
$$

Again the degree sequence is a Fibonacci one except when $\gamma=0$ or $\eta=0$, in which case we have the recursion $d_{n+1}=d_{n-1}+d_{n-2}$, or when $\epsilon_{n}=\gamma_{n} \eta_{n-2}$. In the latter case the degree-growth follows the pattern $d_{n}=0,1,1,2,2,3,3, \ldots$ i.e. a linear growth. Thus we expect this case to be integrable. This is precisely what we found in [13]. Assuming $\eta \neq 0$ we can scale it to $\eta=1$, and thus $\epsilon=\gamma$. The mapping can then be integrated to the homography $\left(x_{n-1}+1\right)\left(x_{n}+1\right)=k_{a x} x_{n-1}$ where $k$ is an integration constant and $a$ is related to $\gamma$ through $\gamma_{n}=-a_{n+1} / a_{n}$. Thus in this case mapping (9) is a discrete derivative of a homographic mapping.

This leads us naturally to the consideration of the generic three-point mapping that can be considered as the discrete derivative of a (discrete) Riccati equation. Let us start from the general homographic mapping which we can write as

$$
A x_{n} x_{n+1}+B x_{n}+C x_{n+1}+D=0 .
$$

where $A, B, C, D$ are linear in some constant quantity $\kappa$. In order to take the discrete derivative we extract the constant $\kappa$ and rewrite (10) as:

$$
\kappa=\frac{\alpha x_{n} x_{n+1}+\beta x_{n}+\gamma x_{n+1}+\delta}{\epsilon x_{n} x_{n+1}+\zeta x_{n}+\eta x_{n} u p+\theta} .
$$

Using the fact that $\kappa$ is a constant, it is now easy to obtain the discrete derivative by downshifting (11) and subtracting it form (11) above. Instead of examining this most general case we concentrate on the forms proposed in [14]. They correspond to the reduction of (11) to the two cases:

$$
\begin{gathered}
\kappa=x_{n+1}+a+\frac{b}{x_{n}} \\
\kappa=\frac{x_{n+1}\left(x_{n}+a\right)}{x_{n}+b}
\end{gathered}
$$

Next we compute the discrete derivatives of (12) and (13). We find:

$$
x_{n+1}=x_{n}+a_{n-1}-a_{n}-\frac{b_{n}}{x_{n}}+\frac{b_{n-1}}{x_{n-1}}
$$


and

$$
x_{n+1}=x_{n} \frac{x_{n-1}+a_{n-1}}{x_{n}+a_{n}} \frac{x_{n}+b_{n}}{x_{n-1}+b_{n-1}}
$$

The study of the degree of growth of (14) and (15) can be performed in a straightforward way. For both mappings we find the sequence $d_{n}=0,1,2,3,4,5,6, \ldots$ i.e. a linear growth just as in the cases of mappings (2), (3) and the integrable subcases of (9). If we substitute $b_{n-1}$ by $c_{n-1}$ in the last term of the rhs of (14) or the denominator of (15) we find $d_{n}=0,1,2,4,8,16, \ldots$ i.e. $d_{n}=2^{n}$ for $n>0$ unless $c=b$. Investigating all the possible ways to curb the growth we find for both (14) and (15) that $c=0$ is also a possibility to bring $d_{3}$ down to 3 . However a detailed analysis of this case shows that for $c=0$ we have $d_{n}=0,1,2,3,5,8,13,21, \ldots$ i.e. a Fibonacci sequence with slower, but still exponential, growth (i.e. ratio $\frac{1+\sqrt{5}}{2}$ instead of 2 ).

One more family of linearisable discrete systems has been studied in detail in [15] and [16]. They are what we called the Gambier mappings which constitute the discretisation of the continuous Gambier equation [17]. The latter is a system of two Riccati's in cascade. In the discrete case the Gambier system is written as two homographic mappings which we write in canonical form as:

$$
\begin{gathered}
y_{n+1}=\frac{a_{n} y_{n}+b_{n}}{y_{n}+1} \\
x_{n+1}=\frac{x_{n} y_{n} / d_{n}+c_{n}^{2}}{x_{n}+d_{n} y_{n}}
\end{gathered}
$$

Eliminating $y$ we can also write the discrete Gambier system as a single three-point mapping for $x$. The study of the degree growth of (16) is straightforward. We start from $x_{0}=r, y_{0}=p / q$ and compute the homogeneous in $p, q$ degree of (16a) and (16b). Since (16a) is a Riccati its degree does not grow i.e. we have $d_{y_{n}}=1$. Given the structure of (16b) we have $d_{x_{n+1}}=d_{x_{n}}+d_{y_{n}}$ and thus $d_{x_{n}}=n$. What is interesting here is that the Gambier mapping exhibits a linear degree-growth independently of the precise values of $a, b, c, d$. The fact that it can be reduced to Riccati's in cascade is enough to guarantee its integrability. On the other hand, if we had asked, (as we have done in [15]) for the possibility to express the solution as an infinite product of matrices,even across singularities, this would have led to constraints on the parameters (which were given in detail in [16]).

In this work we have examined a class of integrable discrete systems (mainly three-point mappings) from the point of view of the degree-growth of the iterates of some initial data. Our study was motivated from the recent works connecting slow-growth and integrability. Our present analysis confirms our previous findings based on the singularity confinement necessary discrete integrability criterion. But what is more important is that a relation between the details of integrability and the degree-growth seems to emerge. In this work 
we have found two main types of degree-growth: zero and linear growth. Zero growth is associated to systems which are linearisable through a reduction to a projective system. Linear growth is characteristic of systems which can be reduced to linear ones although at the price of some more complicated transformations, usually through the existence of some constant of motion or, as in the case of the Gambier mapping, through the solutions of linear equations in cascade. On the other hand, in our study on discrete Painlevé equations and the QRT mapping we found that quadratic growth was the rule. These results are, of course, characteristic of three-point (second-order) mappings and we do not expect the details concerning the precise exponents to carry over to higher-order mappings. Still, we expect the pattern detected here, namely that linearisable mappings lead to slower growth than the nonlinearisable integrable ones, to persist. It could be used for the classification of integrable discrete systems and be a valuable indication as to the precise method of their integration. We intend to return to this point in some future work. 


\section{ACKNowledgements.}

The authors are grateful to J. Fitch who provided them with a new (beta) version of REDUCE without which the calculations presented here would have been impossible. S. Lafortune acknowledges two scholarships: one from FCAR du Québec for his Ph.D. and one from "Programme de Soutien de Cotutelle de Thèse de doctorat du Gouvernement du Québec" for his stay in Paris.

\section{REFERENCES}

[1] B. Grammaticos, A. Ramani, K. M. Tamizhmani, Jour. Phys. A 27 (1994) 559.

[2] G.R.W. Quispel, J.A.G. Roberts and C.J. Thompson, Physica D34 (1989) 183.

[3] B. Grammaticos, F. Nijhoff and A. Ramani, Discrete Painlevé equations, course at the Cargèse 96 summer school on Painlevé equations.

[4] B. Grammaticos, A. Ramani and V.G. Papageorgiou, Phys. Rev. Lett. 67 (1991) 1825.

[5] J. Hietarinta and C.-M. Viallet, Phys. Rev. Lett. 81 (1998) 325.

[6] V.I. Arnold, Bol. Soc. Bras. Mat. 21 (1990) 1.

[7] A.P. Veselov, Comm. Math. Phys. 145 (1992) 181.

[8] M.P. Bellon and C.-M. Viallet, Algebraic Entropy, Comm. Math. Phys. to appear.

[9] Y. Ohta, K.M. Tamizhmani, B. Grammaticos and A. Ramani, Singularity confinement and algebraic entropy: the case of the discrete Painlevé equations, preprint (1999).

[10] M.P. Bellon, J.-M. Maillard and C.-M. Viallet, Phys. Rev. Lett. 67 (1991) 1373.

[11] B. Grammaticos, A. Ramani, Int. J. of Mod. Phys. B 7 (1993) 3551.

[12] A. Ramani, B. Grammaticos, G. Karra, Physica A 180 (1992) 115.

[13] A. Ramani, B. Grammaticos, K.M. Tamizhmani, S. Lafortune, Physica A 252 (1998) 138.

[14] B. Grammaticos, A. Ramani, Meth. and Appl. of An. 4 (1997) 196.

[14] B. Grammaticos and A. Ramani, Physica A 223 (1995) 125.

[15] B. Grammaticos, A. Ramani, S. Lafortune Physica A 253 (1998) 260.

[16] E.L. Ince, Ordinary differential equations, Dover, New York, 1956. 